\title{
Editorial
}

\section{Colombian Applied Linguistics Journal: A consolidated scientific community locally and globally}

With this outstanding group of articles published in the first issue of Volume 21, I would like to close an important professional and personal learning process as the head of the editorial team of the Colombian Applied Linguistics Journal. I feel grateful to my institution, Universidad Distrital Francisco José de Caldas, for the professional support received during the last 10 years by hiring a technical team through the Center for Research and Scientific Development (CIDC) to assist the arduous job of the editor. Moreover, the scientific community of the Colombian Applied Linguistics Journal was also fully supported by other national and international communities of scholars who are committed to the same high quality standards in publication or have been in the field for a longer trajectory. To those thousands of authors, peer evaluators, colleagues in the scientific and editorial boards who have accompanied the editor for 20 years I am forever grateful. It was your professional judgment and ethical practice and our rigorous and ethical editorial practice that permitted to position the Colombian Applied Linguistics Journal as a solid scientific publication and a valuable source for teacher educators and researchers in Latin America and other parts of the globe where CALJ has a wide audience.

I have personally and professionally learned a great deal about what it takes to create, sustain and empower academic communities through the commitment of publishing scientific articles. Through various stages in the editing process I dealt with authors, evaluators, scientific committees, assistants, proofreaders, indexes, SIRes and Publindex. I learned to listen and understand different points of view always considering what was best for the readership. As an author myself, I always cared about the expectations, feelings, encouraging or discouraging moments that authors experienced when facing writing for publication. It was important, as editor, to explain the criteria and editing workflow of the articles to each one of the authors. Thus, annually in the launching event of the first issue of the journal we informed the audience about the underpinnings of the process of receiving, evaluating, editing and publishing the articles. I have always valued the global network I constructed through professional but spontaneous invitations to know our academic journal. My encounters with colleagues in conferences whose academic work signified a contribution became a possibility for an invitation to publish in our scientific journal or to participate as an evaluator for a particular article. These and many other activities are the everyday dynamics of maintaining a community of practice in the editorial world.

This issue offers seven research articles and two book reviews. The first article deals with topics related to using cultural content in the English lessons to help undergraduate students develop meaningful communication through cultural knowledge. The authors, Oviedo, H., \& Álvarez, H. (2019) explored undergraduate students' responsiveness to cultural issues and activities in their process of learning English. The second article by Cuesta Medina L., Alvarez Ayure C.P., Cadena Aguilar, A., Jiménez Bonilla, M. S., Maldonado Chacón, P., \& Morales Pulido, V. (2019) deals with the value of reflection in Language Teaching and Learning with in-service language teachers pursuing a graduate degree. The results of their study unveil the difficulties 
that in-service teachers have in their cognitive, metacognitive and linguistic domains. The authors report teachers' inability to self-assess, self-monitor, and self-evaluate their academic performance. They consider that participants could be assisted in the development of selfregulatory skills as they grow in their professional continuum to face the challenges set by their teaching contexts while they bridge their learning gaps.

A third contribution addresses foreign language teachers' identity. Brazilian and Chilean teacher educators and researchers Archanjo, Barahona \& Finardi (2019) focused on three main issues: emerging identities, the role of foreign language proficiency to study how foreign language pre-service teachers develop and ways to conceptualize their teacher identity in three teacher education programs in Brazil and Chile. Their results suggest that FL teacher identity is shaped by notions of legitimization of the teacher's role and language proficiency.

Luis Ricardo Rojas and Jenifer Rueda at a public university in the South of Colombia, introduced Embera Chamí students to English learning through Task and Project-Based Learning. They developed a project with students that would raise awareness of drugs' harmful effects and at the same time learn about the kind of English they need to use to sell their crafts in different fairs around the country.

The fifth article by Ximena Paola Buendía and Diego Fernando Macías provides a review of 25 empirical studies on professional development of English language teachers in Colombia. The authors suggest that there is a need to move from traditional master-apprentice, contentoriented, teacher-centered models of professional development towards initiatives that allow teachers to critically analyze their particular context and needs, and devise their own local alternatives so that they can become more active agents of their own process of change.

Mauricio Mancipe and Cynthia Marcela Ramírez in their article "The role of language in elaborating explanations in the science class in bilingual contexts through CLIL", analyze the role of language in teaching from two perspectives: communicative and explanatory. The authors carried out a pedagogical implementation with students using the CLIL approach and their findings report a close link between the L2 proficiency, and the depth of the explanations elaborated by the students. They considered that the implementation re-dimensioned the content perspective applied by teachers when using the CLIL, placing bilingualism in the science classes as a medium that fosters communicative and explanatory processes by nurturing different cognitive linguistic abilities.

Lastly, Elizabeth Flores-Salgado y Michael T. Witten do a comparative study of Mexican and Irish compliment responses and they find that social factors (social distance, social power, gender, and the topic of the compliment) in both the Mexican as in the and Irish society seem to be crucial parameters in the formulation and acceptance or rejection of a compliment.

Two book reviews are also part of this issue. The first by Laryssa Paulino de Queiroz Sousa on Posthumanism in applied linguistics; and the second by Ómar Garzón on quality standards and unequal conditions in initial teacher education in Colombia. They add to the professional discussion on the existing challenges in teacher education within the field of applied linguistics. At this point and having introduced the current issue, I would like to recall some of the themes from previous editorial articles that have been significant in the construction of this scientific community. 
a. The importance of raising awareness about the local appropriation of languages by using the local contexts for teaching and researching. This way, teachers are closing the existing gap between the foreign language and the students' knowledge and realities.

"The teaching of English as a foreign language in our Latin American contexts requires that all educators become familiar with and actively address the needs of diverse learners; participate in the social practices that constitute the EFL professional field, and implement critical approaches that integrate content, local contexts and language education so that new educational challenges can be faced having the needs of the communities of students in mind"

Clavijo, A. (2015). January - June 2015. Vol. 17 • Number 1 pp. 4-10.

b) Another recent theme is the status of local teachers of English in the unequal treatment of native and nonnative speakers of English.

The preference for native speaker teachers of English (NS) over Non-native speakers (NNS) to work in public and private schools in Latin American countries places teachers at a disadvantage and "increases the loss of public trust in local teachers and in university-based programs that train English teachers" (Llurda E González, 2016:104).

Clavijo, A. (2017). Vol 19. Number 2. 161-164.

c) Belonging to a Community of Research Practice

Regarding my professional concern about the writing and publishing dimensions of research productivity and how writers' understanding of the nature of journal article writing can be developed, I propose to develop communities of writing practice by means of communities of research practice at our higher education institutions. Communities of practice, as Wenger (1998) puts it, are groups of people who share a concern or a passion for something they do and learn how to do it better as they interact regularly. Thus, if we work on creating healthy productive environments within our communities of research in higher education institutions we should possibly facilitate and successfully develop our research and publication agendas.

Clavijo, A. (2015). July - December 2015. Vol. 17• Number 2 pp. 175-17

\section{d) Colombian Applied Linguistics reaches 20 years of publication}

With the increasing impact of technologies in the sociosemiotic landscape of our contemporary communities one wonders about the directions our professional field may take when faced with new generations of learners that live on and from the screen and how to educate teachers that can use these new ways to communicate in the service of a critical education. Texting, messaging, Skyping, whatsapping, voicing, videoing and more are communicative practices that engage a new kind of social networking which our pedagogies need to incorporate. Yet, in Latin America the inclusion of these practices should align with larger educational goals for social justice, equality, respect for diversity and democracy that orient modern societies and that call for critical approaches in applied linguistics.

Clavijo, A. (2018). Colombian Applied Linguistics Vol. 20 • Number 1 pp. 11-24

The Colombian Applied Linguistics Journal has been steered by some of these ideas and principles in these years I have been at the helm. They have been fundamental in helping the journal gained the recognition of the academic community and, I hope, in ushering the way to a promissory future. 


\section{References}

Clavijo-Olarte, A. (2015). Research tendencies in the teaching of English as a foreign language. Colomb. Appl. Linguist. J.17(1), 5-10. https://doi.org/10.14483/22487085.9006

Clavijo-Olarte, A. (2015). Belonging to a Community of Research Practice. Colomb. Appl. Linguist. J., 17(2), 175-178. https://doi.org/10.14483/http://dx.doi.org/10.14483/udistrital.jour.calj.2015.2.a00

Clavijo-Olarte, A. (2017). Exploring oral discourse development in the EFL classroom: perspectives from Ecuador, Honduras, Chile \& Colombia. Colomb. Appl. Linguist. J., 19(2), 161-164. https:// doi.org/10.14483/22487085.12415

Clavijo Olarte, A. (2018). Colombian Applied Linguistics reaches 20 years of publication. Colomb. Appl. Linguist. J., 20(1), 4-7. https://doi.org/10.14483/22487085.13193

González, A. E Llurda, E. (2016). Bilingualism and globalization in Latin America: fertile ground for native-speakerism. In F. Copland, S. Garton, \& S. Mann. (Eds.). LETs and NESTs: Voices, Views and Vignettes. London: British Council.

Wenger, E. (1998). Communities of practice: learning meaning and identity. Cambridge: CUP.

Amparo Clavijo-Olarte PhD

EDITOR 


\section{Editorial}

\section{La Revista Colombiana de Lingüística Aplicada una comunidad científica consolidada local y globalmente}

Con este destacado conjunto de artículos publicados en el primer número del volumen 21, finalizo un importante proceso de aprendizaje profesional y personal como cabeza del equipo editorial de la revista Colombian Applied Linguistics. Me siento agradecida con mi institución, Universidad Distrital Francisco José de Caldas, por el apoyo personal recibido durante los últimos 10 años, con la contratación de un equipo técnico a través del Centro de Investigaciones y Desarrollo Científico (CIDC), para asistir la ardua labor del editor. Adicionalmente, La comunidad científica de la revista ha tenido el apoyo total de otras comunidades nacionales e internacionales de académicos que tienen los mismos altos niveles de calidad en publicaciones o que han estado en el campo durante un mayor tiempo. A todos aquellos autores, pares evaluadores y colegas en los comités científico y editorial que han acompañado al editor por 20 años, estoy eternamente agradecida. Fue su juicio profesional y práctica ética, así como nuestro riguroso y ético ejercicio editorial, lo que permitió posicionar a Colombian Applied Linguistics como una publicación científica sólida y como un recurso valioso para educadores de docentes e investigadores en Latinoamérica y en otras partes del mundo donde CALJ tiene una amplia audiencia.

Personal y profesionalmente, he aprendido bastante sobre lo que se requiere para crear, sostener y empoderar comunidades académicas a través de la publicación de artículos científicos. A través de las distintas fases del proceso editorial, traté con autores, evaluadores, comités científicos, asistentes, correctores de estilo, índices, SIRes y con Publindex. Aprendí a escuchar y entender distintos puntos de vista, siempre pensando en lo mejor para la audiencia. Gracias a mi experiencia como autora, siempre tuve presentes las expectativas, sentimientos, y momentos alentadores y desalentadores que experimentan los autores cuando se encuentran frente a la tarea de escribir para publicar. Era importante, como editora, explicar los criterios y el flujo de trabajo editorial de los artículos a cada uno de los autores. Por eso, cada año en el lanzamiento del primer número de la publicación, informamos a la audiencia sobre los aspectos subyacentes al proceso de recibir, evaluar, editar y publicar los artículos. Siempre he tenido un gran aprecio hacia la red global de expertos que construí a través de invitaciones profesionales, pero espontáneas, para dar a conocer nuestra revista académica. Mis encuentros en conferencias con colegas cuyo trabajo académico podría significar una contribución, se convirtieron en una posibilidad para hacer una invitación a publicar en nuestra revista, o a participar como evaluadores de algún artículo en particular. Estas y muchas otras actividades son dinámicas cotidianas del mantenimiento de una comunidad en el mundo editorial.

Este número ofrece siete artículos y 2 reseñas de libros. El primer artículo trata sobre los temas relacionados con el uso de contenido cultural en lecciones de inglés para ayudar a estudiantes de pregrado a desarrollar una comunicación significativa a través del conocimiento cultural. Los autores, Oviedo, H., \& Álvarez, H. (2019), exploraron la sensibilidad de los estudiantes frente a aspectos y actividades culturales en su proceso de aprendizaje de la lengua inglesa. El segundo artículo, de Cuesta Medina L., Álvarez Ayure C.P., Cadena Aguilar, A., Jiménez Bonilla, M. S., Maldonado Chacón, P., \& Morales Pulido, V. (2019), se enfoca en el valor de la reflexión sobre la enseñanza y el aprendizaje de idiomas con profesores de idiomas en servicio que persiguen un título de pregrado. Los resultados de su estudio revelan las dificultades que 
los profesores en servicio presentan en los dominios cognitivo, metacognitivo y lingüístico. Los autores reportan la dificultad de los profesores para autoevaluar y auto monitorear su desempeño académico. Consideran que los participantes podrían recibir apoyo en el desarrollo de habilidades autorregulatorias, a medida que se desarrollan profesionalmente, para encarar los retos impuestos por los contextos de enseñanza mientras se llenan los vacíos de aprendizaje.

Una tercera contribución trata sobre la identidad de los profesores de lengua extranjera. Las investigadoras brasileñas y chilenas Archanjo, Barahona \& Finardi (2019) se centraron en tres problemas principales: las entidades emergentes, el rol de la proficiencia en lengua extranjera para estudiar cómo los docentes en formación en lengua extranjera en tres programas de formación en Brasil y Chile se desarrollan profesionalmente y conceptualizan su identidad como docentes. Los resultados sugieren que la identidad de los profesores de lengua extranjera está moldeada por nociones de legitimización del rol de los profesores y por la proficiencia en un idioma.

Luis Ricardo Rojas y Jenifer Rueda introdujeron a los estudiantes Embera Chamí de una universidad pública del sur de Colombia al aprendizaje de inglés basado en tareas y proyectos. Desarrollaron un proyecto con los estudiantes que podría aumentar la conciencia de los efectos nocivos de las drogas y, al mismo tiempo, llevar al aprendizaje sobre el tipo de inglés necesario para vender artesanías en distintas ferias a través del país.

El quinto artículo, de Ximena Paola Buendía and Diego Fernando Macías, proporciona Una revisión de 25 estudios empíricos acerca del desarrollo profesional de profesores de lengua inglesa en Colombia. Los autores sugieren que es necesario salir de los modelos tradicionales de desarrollo profesional de maestro-aprendiz, que son orientados al contenido y centrados en el profesor, con el fin de llegar a iniciativas que permitan a los profesores analizar críticamente su contexto particular y sus necesidades, y desarrollar sus propias alternativas locales para poder convertirse en agentes más activos de su propio proceso de cambio.

Mauricio Mancipe E Cynthia Marcela Ramírez, en su artículo "El papel del lenguaje en la construcción de explicaciones en la clase de ciencias en contextos bilingües a través del enfoque CLIL", analizan el papel del idioma en la enseñanza desde dos perspectivas: comunicativa y explicativa. Los autores llevaron a cabo una implementación pedagógica con los estudiantes usando la aproximación a CLIL, y sus hallazgos reportan un vínculo cercano entre la proficiencia en L2 y la profundidad de las explicaciones elaboradas por los estudiantes. Consideran que la implementación redimensionó la perspectiva sobre el contenido aplicada por los profesores cuando usaron CLIL, lo que sitúa el bilingüismo en las clases de ciencia como un medio que impulsa los procesos comunicativos y explicativos a través del desarrollo de distintas habilidades lingüísticas y cognitivas.

Finalmente, Elizabeth Flores-Salgadoy Michael T. Witten quienes realizaron un estudio comparativo de las respuestas a cumplidos producidas por 60 hablantes nativos de español de México y 60 hablantes nativos de inglés de Irlanda, encontraron que los factores sociales (distancia social, poder social, género y el tema del cumplido) en la sociedad mexicana y en la irlandesa parecen ser parámetros cruciales en la formulación y aceptación o rechazo de un cumplido.

También hacen parte de este número dos reseñas de libros. La primera es escrita por Laryssa Paulino de Queiroz Sousa, acerca del posthumanismo en la lingüística aplicada; y la segunda, por Omar Garzón, acerca de los estándares de calidad y las condiciones desiguales en la formación inicial de docentes en Colombia. Estos textos contribuyen a la discusión profesional 
de los retos existentes en la formación de docentes dentro del campo de la lingüística aplicada. En este punto, me gustaría hacer referencia a algunos de los temas de los artículos editoriales previos que han sido significativos en la construcción de esta comunidad científica.

a) La importancia de aumentar la conciencia acerca de la apropiación local de las lenguas, por medio del uso de los contextos locales para enseñar e investigar. Así, los profesores cierran la brecha existente entre la lengua extranjera y el conocimiento y las realidades locales de los estudiantes.

"la enseñanza de inglés como lengua extranjera en nuestros contextos latinoamericanos requiere que todos los educadores se familiaricen y aborden las necesidades de la diversidad de estudiantes en sus aulas; participen en las prácticas sociales que constituyen el campo profesional de la Enseñanza del Inglés como lengua extranjera, e implementen enfoques críticos que integren contenidos, contextos locales y la enseñanza de las lenguas para que los nuevos retos educativos pueden ser enfrentados teniendo en cuenta las necesidades de las comunidades de estudiantes." Clavijo, A. (2015). Enero-Junio 2015. Vol. 17 • Número 1 pp. 4-10.

b) Otro tema reciente es el estatus de los docentes locales de inglés en el tratamiento desigual de hablantes nativos y no nativos de inglés.

"La preferencia por maestros hablantes nativos del inglés que es mayor a la de profesores hablantes no nativos para trabajar en escuelas públicas y privadas en países latinoamericanos, coloca a los maestros hablantes no nativos del inglés en desventaja y "aumenta la pérdida de confianza pública en maestros locales y en los programas de formación de profesores de inglés en universidades" (Llurda \& González, 2016: 104).”

Clavijo, A. (2017). Vol 19. Número 2. 161-164.

c) Pertenecer a una comunidad de práctica investigativa.

Propongo desarrollar comunidades de prácticas de la escritura por medio de comunidades de práctica de la investigación en nuestras instituciones de educación superior. Las comunidades de práctica, como Wenger (1998) lo dice, son grupos de gente quienes comparten una preocupación o una pasión por algo que ellos hacen y aprenden cómo hacerlo mejor, ya que interactúan regularmente. Por lo tanto, si trabajamos en la creación de ambientes productivos sanos dentro en nuestras comunidades de investigación en la educación superior, nosotros deberíamos posiblemente facilitar y desarrollar con éxito nuestras agendas de investigación y publicación.

Clavijo, A. (2015). Julio - Diciembre 2015. Vol. 17• Número 2 pp. 175-17

d) Colombian Applied Linguistics Journal cumple 20 años de publicación.

Con el creciente impacto de las tecnologías en el paisaje sociosemiótico de nuestras comunidades contemporáneas, uno se pregunta sobre las direcciones que puede tomar nuestro campo profesional cuando se enfrenta a nuevas generaciones de estudiantes que viven en y desde la pantalla y cómo educar a los maestros que pueden usar estas nuevas formas de comunicarse al servicio de una educación crítica. Mensajes de texto, mensajería, Skype, WhatsApp, voz, video y más son prácticas comunicativas que involucran un nuevo tipo de redes sociales que nuestras pedagogías necesitan incorporar. Sin embargo, en América Latina, la inclusión de estas prácticas debería alinearse con objetivos educativos más amplios de justicia social, igualdad, respeto a la diversidad y democracia que orientan a las sociedades modernas y que requieren enfoques críticos en la lingüística aplicada. 
La Revista Colombian Applied Linguistics ha sido guiada por algunas de estas ideas y principios durante los años en los que he estado al mando. Estas han sido fundamentales para ayudar a la revista a ganar el reconocimiento de la comunidad académica y, espero, para abrir el camino hacia un futuro prometedor.

\section{Referencias}

Clavijo-Olarte, A. (2015). Research tendencies in the teaching of English as a foreign language. Colomb. Appl. Linguist. J.17(1), 5-10. https://doi.org/10.14483/22487085.9006

Clavijo-Olarte, A. (2015). Belonging to a Community of Research Practice. Colomb. Appl. Linguist. J., 17(2), 175-178. https://doi.org/10.14483/http://dx.doi.org/10.14483/udistrital.jour.calj.2015.2.a00

Clavijo-Olarte, A. (2017). Exploring oral discourse development in the EFL classroom: perspectives from Ecuador, Honduras, Chile \& Colombia. Colomb. Appl. Linguist. J., 19(2), 161-164. https://doi. org/10.14483/22487085.12415

Clavijo Olarte, A. (2018). Colombian Applied Linguistics reaches 20 years of publication. Colomb. Appl. Linguist. J., 20(1), 4-7. https://doi.org/10.14483/22487085.13193

González, A. \& Llurda, E. (2016). Bilingualism and globalization in Latin America: fertile ground for native-speakerism. In F. Copland, S. Garton, \& S. Mann. (Eds.). LETs and NESTs: Voices, Views and Vignettes. London: British Council.

Wenger, E. (1998). Communities of practice: learning meaning and identity. Cambridge: CUP.

Amparo Clavijo-Olarte PhD EDITOR 